\title{
Adhesive and cohesive force matters in deformable batteries
}

\author{
Guojin Liang ${ }^{1}$, Jiaxiong Zhu' ${ }^{1}$, Ao Chen ${ }^{1}$, Qi Yang ${ }^{2}$ and Chunyi Zhi ${ }^{1,2 \bowtie}$
}

Mechanical stability of flexible batteries is the guarantee for delivering stable performance. The interacting external and inner forces determine it.

npj Flexible Electronics (2021)5:27; https://doi.org/10.1038/s41528-021-00124-w

Deformable battery is one core component as a power supply in wearable electronic systems, where its mechanical stability weighs equal significance compared to electrochemical performance. Thanks to the past 40 years' efforts in developing highperformance battery systems, generally to be applied in static scenarios, materials in each battery components are electrochemically mature and ready enough, e.g., commercial Li-ion and $\mathrm{Zn}$ ion batteries, to be transferred into deformable configurations ${ }^{1,2}$. Yet, the highly desired deformable batteries are not widely commercialized, dominantly due to the status quo of their mechanical performance might not be competent for practical applications. Specifically, considering the inevitable and various mechanical manipulations under service, mechanical dysfunctionalities of deformable batteries are difficult to be predicted and avoided. In this regard, guaranteeing deformable batteries with long-term mechanical stability would be more challenging compared to endowing statically electrochemical stability ${ }^{3,4}$.

Looking back to the progress of deformable batteries during the last decade, the mainstream efforts were devoted to endowing mechanical deformability (flexibility and/or stretchability) to different battery systems through configurational and material designs ${ }^{5-7}$, such as chain-like, origami, and spine-inspired structures and/or merging rigid material into elastic substrates, where such configurational regulation strategies can redistribute the applied forces to minimize the force magnitude on specific battery region. However, the inevitable structural variations from the initially equilibrium states, either in single layer or multilayers, might occur to some extent, which would lead to performance degradations ${ }^{8}$. One workable solution is to build up mechanically durable battery configurations ${ }^{9,10}$. However, when checking the device fabrication protocols, different functional layers are simply stacked together without special consideration on a design criteria to construct mechanically robust batteries.

There are diverse sorts of forces applied on deformable batteries, such as bending, twisting, stretching, compressing forces that different structural deformations would appear accordingly. Herein, the most frequently applied bending force is analyzed as an exemplifying model force to clearly elaborate battery structure evolutions subjected to external forces. In this Comment, we first analyze the possible structure evolutions correlating to batteries performance degradations, and then the improving strategies from material aspects are proposed to solve these issues.

\section{HOW BATTERY DEFORMS UNDER BENDING FORCE}

Considering the prototypical battery configurations are constructed in a layer-stacking structure, we start with the single component layer to study the structural evolutions throughout bending force. A curved shape would be gradually formed once the bending forces are applied, where the tensile stress will concentrate on the convex surface and compressive stress at the concave surface (Fig. 1a). If the external force does not exceed the yielding point, it would recover back to its initially equilibrium state after the force is removed. In another word, the deformation is totally reversible as elastic deformation. In counter case, once the plastic deformation is triggered, it could cause permanent structural changes without recovering back to initial states ${ }^{9}$. Mesoscopic curve-shaped crease and/or microscopic domain cracks would appear based on a brittle fracture mechanism to disperse the overloaded strain (Fig. 1a). Such structural changes will directly result in electrochemical performance instabilities and degradations. Regarding the cracks after deformation, one possible solution is to apply self-healing binders to recover back to their initial state.

Moving forward to the multiple-layered structure, we imitate the deformable battery under realistic applications by modeling all component layers by stacking in the sequence of the encapsulation, current collector for cathode, cathode, separator, anode, current collector for anode, and encapsulation layer. When observing the deformation manners of each single layer in the multiple-layered structure, it obeys the same deformation behaviors of the separated single layer as illustrated in Fig. 1a. When analyzing the structure deformation manners of multiple layers, there are two forms of bending forces as one-end and twoends fixed bending forces. Firstly, under one-end fixed bending forces, the interlayer slippages will occur between adjoining layers at the unfixed end (Fig. 1b). Secondly, regarding the two-ends fixed bending, the interlayers will detach to cause layer delaminations at the strain concentrated spot (Fig. 1c). In both cases, under the premise that no plastic deformations occur in all layers, the batteries can recover back to their original structures after the bending forces are removed, where the slippages and delaminations are transient state during force manipulations. Therefore, battery performance might be unstable during the bending process, but recovered back after reversible deformation. Otherwise, irreversible plastic deformations retain with permanent slippages and delaminations in interlayers, and elongations and creases in some layers, resulting in unrecovered performance degradations. When coupled with the flammable and aggressive electrolytes, temperature-rising or even fire-catching issues are possible to occur during mechanical manipulations. It should be emphasized that no matter the reversible or irreversible 
a

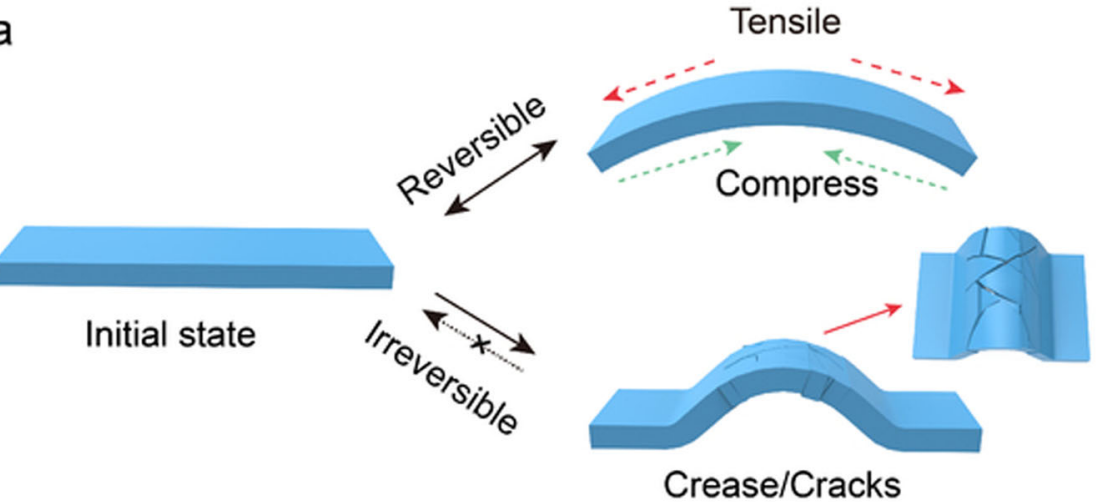

b

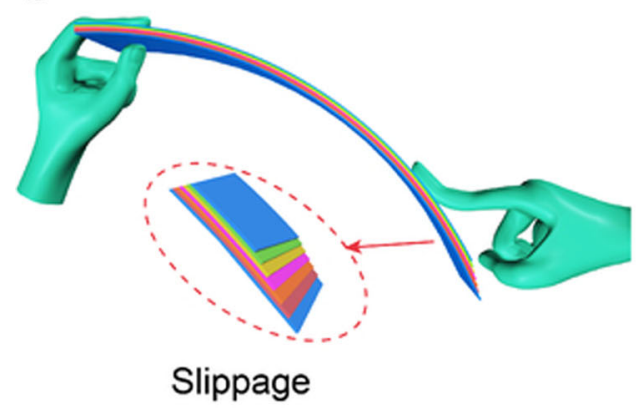

C

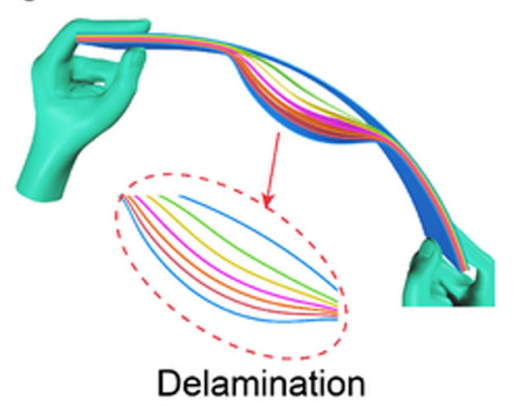

Fig. 1 Schematics of structural evolutions of single layer and full battery device under bending force. a Initial state and final state of deformable single layer throughout reversible elastic deformation and irreversible plastic deformation. b In one-end fixed bending of deformable batteries, slippage transiently occurs during elastic deformation and permanently exists after plastic deformation. c In two-ends fixed bending of deformable batteries, delaminations transiently occur during elastic deformation and permanently exist after plastic deformation.

deformations occurring, the structural variations from initial states are correlated to performance instability and/or degradation.

\section{HOW TO IMPROVE COHESIVE AND ADHESIVE FORCES}

From material aspects, the above-discussed structural variations can be suppressed or even eliminated by regulating materials to construct mechanically durable battery structures. It is primary to perceive the exact materials providing mechanical properties in each layer. There are two different forces as cohesive force within the single layer and adhesive forces between multiple layers to preserve their original structures, i.e., resisting the deformations under external force. Specifically, it raises a question from material aspects that how to enhance the cohesion and adhesion inside deformable batteries. Since there are different materials involved in electrochemical behaviors, functional layers can be classified as the single component layer and composite layer. Regarding the single component layers, they are the current collectors as $\mathrm{Cu}$ for anode, Al for cathode, and separator as polypropylene, of which the yield strengths and mechanical moduli are generally determined. As for the cathode and anode composite layers, they are constituted with composite materials such as electrochemical active materials, polymer binders, and conductive additives. The cohesion inside the electrode layers is largely determined by the polymeric binder materials, where the binding force is based on weak van der Waals interactions, e.g., the common binder as polyvinylidene difluoride (PVDF), easily resulting in mechanical cracks in the electrode layer during bending ${ }^{11}$. Therefore, the cohesion improving strategy is to develop robust elastic binders in electrode layer processing high elastic modulus and resilience, where the polymer-network wrapping around electrochemically active particles (Fig. 2a-i). The zoomed-in layer structure in the stretched state, as demonstrated in Fig. 2a-ii, can be recovered back to the initial state after the external stress is removed due to the elastic mechanism of the binder. In theory, the polymer binder under deforming manners should follow nonlinear stiffening manners with high elasticity and smaller stress accumulation during the strain increasing process, improving the yielding strength of the single-layer structure ${ }^{12}$. In addition, high fracture toughness is also required to avoid further crack propagation after the formation of micro-defects.

For the multilayer structure under bending, introducing robust binders to enable strong interlayer adhesion between adjoining layers is an effective strategy to preserve its original structure after deformation. As illustrated in Fig. 2a-iii, the above-discussed permanent deformations as slippage and delamination can be avoided to large extent due to the large resilience of the interlayer adhesion. However, it seems intuitively difficult to build up strong adhesive bonding between two adjoining heterogeneous surfaces. Taking the interface between the electrode layer and current collector layer as an example, the conventional adhesion is realized by binders, e.g., PVDF, based on weak interactions to enable the electrode layer adhering onto metal current collectors. However, the design principles to build up strong adhesives based on covalent bonds at interfaces are still elusive in the field of deformable batteries, especially by considering the interfaces are generally wet due to the existence of liquid electrolytes, adding more difficulties to introduce stable adhesives at interface ${ }^{13}$. Bao et al. have developed one possible solution by applying polymer electrolyte containing supramolecular binders to enable enhanced adhesion at the electrode-electrolyte interface throughout a dynamic bonding ${ }^{3}$. Overall, the interface should be elastic and tough to ensure a good recovery after the interlayer sliding. Thanks to the pioneering works on building topological adhesive 


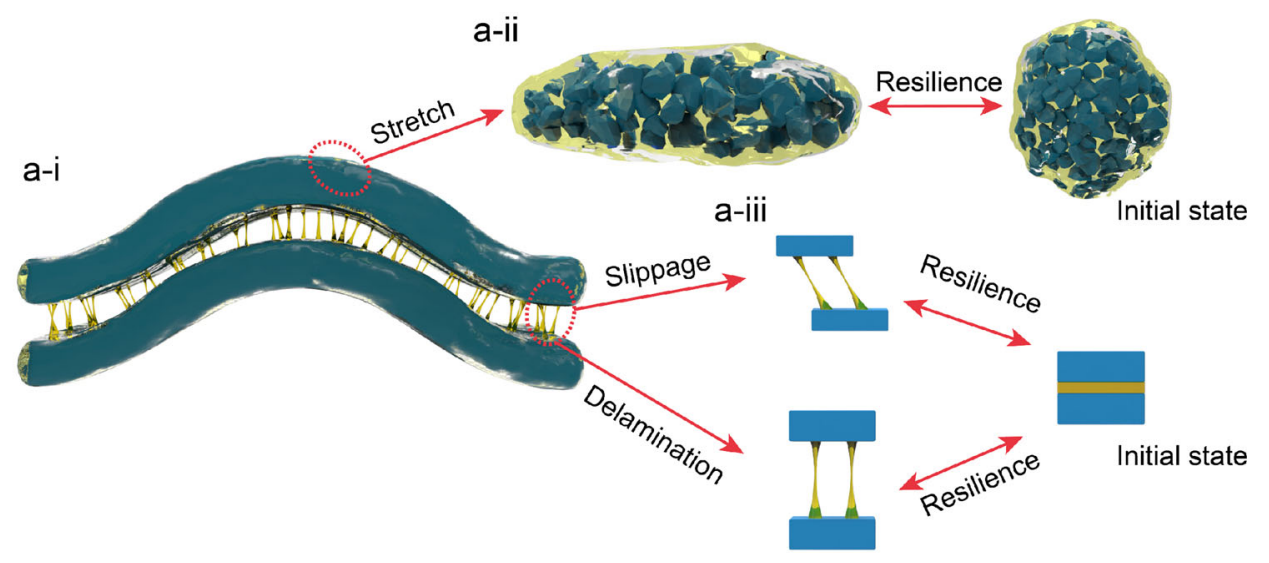

b

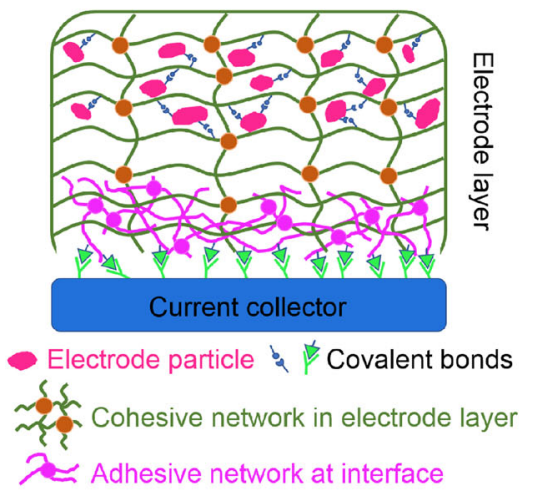

c-i

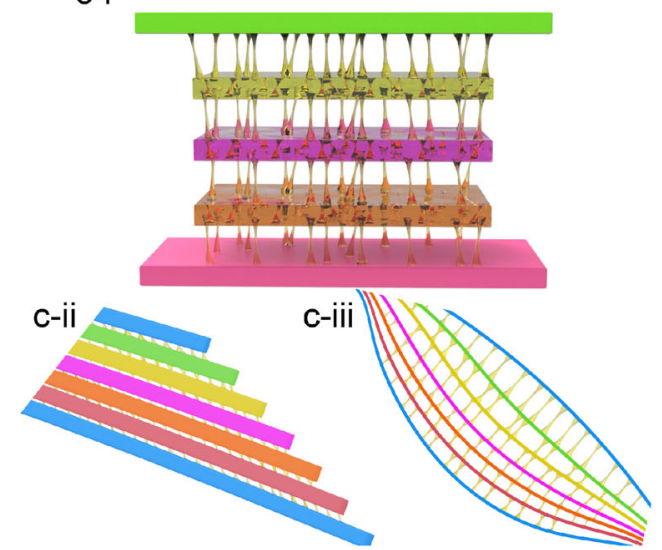

Fig. 2 Schematic illustration of cohesion and adhesion existed in the deformable battery. a-i Applying robust binders to improve cohesive force in electrode layer, where the polymeric network of binders tightly wrapping around electrode particles. Internal cohesion (a-ii) and interlayer adhesion (a-iii) with strong resilience to preserve original structure after deformation. $\mathbf{b}$ Illustrations on the bonding chemistry between the exemplified two-layered configurations, i.e., electrode layer and current collector. Cohesive network is originated from the binders inside the electrode layer and the adhesive layer is based on the design principle of topological adhesion. c-i The schematic of all-inone configuration with cohesion and adhesion by the same binder, forming chemically adhesive bonds throughout the battery device. Adhesions to inhibit the deformation of slippage (c-i) and delamination (c-ii) during bending.

forces based on covalent bonds at interface of two-layered configurations, i.e., water-containing polymeric hydrogel and different substrates, it sheds light on introducing analogous strategies into multiple-layered configurations of deformable battery to apply foreign polymer network as a topological adhesive to sparsely penetrate into two adjoining layers ${ }^{13,14}$. Get inspired by this principle, topological adhesion at the interface can be established on strong chemical bonds, as schemed in Fig. $2 \mathrm{~b}$. It is ideal to apply the single adhesive to every individual layer and the interfaces throughout all different layers to simultaneously solve the adhesion problems, and the ideal all-in-one configuration is realized (Fig. 2c-i), where the adhesives can enable resilience to recover the transient slippage and delamination issues proposed above (Fig. 2c-ii, c-iii). Regarding the basic mechanical requirements, the adhesives should possess high elastic modulus and resilience, which respectively correlated to durability to undertake large deformation and resilience to recover back to the initial state. Of note, strong adhesive force might promote the hardness inside the individual layer and/or at the interface, while the hardness is the countering property to the softness of the deformable device. Therefore, concerns on the softness of the batteries are needed when enhancing adhesion forces. Lastly, the interfacial adhesion between the electrode layer and electrolyte layer is exemplified above as optimizing guidance for other component layers, especially for the packing layer that detaching failures usually occur between the packaging/encapsulation layer and the inner electrochemically active components.

\section{PERSPECTIVE ON MECHANICALLY STABLE BATTERIES}

This comment has discussed the emerging mechanical issues of deformable batteries by analyzing the structural evolutions correlated to performance degradations, moving from single layer to multilayers in one full battery configuration. There are transient and permanent structural variations throughout the bending processes, such as cracks, slippage, and delamination. Regarding the solving strategies from material aspects, it is the binder material that should be well designed and introduced to construct strong cohesive force inside every single layer and strong adhesive force at interfaces of adjoining layers ${ }^{9,15}$. However, binders are generally developed to improve the performance, especially in the electrode layers, for static battery configurations. When considering the deformable batteries under dynamic external forces, cases become more complex to simultaneously ensure stable performance from mechanical and electrochemical dimensions, while it seems more challenging to consider every structural spot to preserve them unvaried. Organic materials can serve as components in all the functional layers, such as electrode, electrolyte, and encapsulation layers, and provide a good solution to the deformability, where their interfacial bonding to other materials can be tuned ${ }^{16,17}$. It should be emphasized that 
electrochemical performance, especially the energy density and cyclic stability, is the primary parameter towards commercially practical applications, therefore only agreeable compromises in electrochemical performance are acceptable when optimizing mechanical performance. Of note, there are many other concerns to be further addressed, for example, whether the liquid-state electrolytes affect the stability of interfacial adhesion, whether the modifications on electrode materials would accelerate the degradation of electrochemical performance, how other external forces, e.g., twisting, stretching and compressing, would affect the structural stability. Unluckily, workable binders to satisfy the above-discussed criteria are limited at the current stage.

For the future optimization on the mechanical performance of deformable batteries, there are two synergic directions deserving more efforts in following research as (1) designing suitable binders with superior elasticity and fracture toughness to counteract the deforming tendency of battery structure, (2) developing optimized battery configurations to dissipate and dilute external forces applied onto battery units. In this regard, designing the binders and building up robust cohesion and adhesion rely on polymer chemistry and mechanic science, where electrochemistry should also be counted in. It is a call for actions to enhance cohesive and adhesive forces when designing deformable battery devices.

Received: 30 April 2021; Accepted: 7 September 2021; Published online: 28 September 2021

\section{REFERENCES}

1. Chang, J., Huang, Q. \& Zheng, Z. A figure of merit for flexible batteries. Joule 4, 1346-1349 (2020).

2. Li, H. et al. An extremely safe and wearable solid-state zinc ion battery based on a hierarchical structured polymer electrolyte. Energy Environ. Sci. 11, 941-951 (2018).

3. Mackanic, D. G. et al. Decoupling of mechanical properties and ionic conductivity in supramolecular lithium ion conductors. Nat. Commun. 10, 5384 (2019).

4. Li, H., Tang, Z., Liu, Z. \& Zhi, C. Evaluating flexibility and wearability of fexible energy storage devices. Joule 3, 613-619 (2019).

5. Qian, G. et al. Designing flexible lithium-ion batteries by structural engineering. ACS Energy Lett. 4, 690-701 (2019).

6. Mackanic, D. G., Kao, M. \& Bao, Z. Enabling deformable and stretchable batteries. Adv. Energy Mater. 10, 2001424 (2020).

7. Wang, Z. et al. Fully transient stretchable fruit-based battery as safe and environmentally friendly power source for wearable electronics. EcoMat 3, e12073 (2021).

8. Brenneman, J., Tansel, D. Z., Fedder, G. K. \& Panat, R. Interfacial delamination and delamination mechanism maps for 3D printed flexible electrical interconnects. Extrem. Mech. Lett. 43, 101199 (2021).

9. Lopez, J., Mackanic, D. G., Cui, Y. \& Bao, Z. Designing polymers for advanced battery chemistries. Nat. Rev. Mater. 4, 312-330 (2019).

10. Chang, J., Huang, Q., Gao, Y., Zheng, Z. Pathways of developing high-energydensity flexible lithium batteries. Adv. Mater. 2004419 https://doi.org/10.1002/ adma.202004419 (2021).
11. Carretero-González, J., Montero, J. \& López-Manchado, M. A. In Smart Polymers and their Applications 2nd edn (eds. Aguilar, M. R. \& San Román, J.) 651-669 (Woodhead Publishing, 2019).

12. Choi, S., Kwon, T.-w, Coskun, A. \& Choi, J. W. Highly elastic binders integrating polyrotaxanes for silicon microparticle anodes in lithium ion batteries. Science 357, 279-283 (2017).

13. Yang, J., Bai, R., Chen, B. \& Suo, Z. Hydrogel adhesion: a supramolecular synergy of chemistry, topology, and mechanics. Adv. Funct. Mater. 30, 1901693 (2020).

14. Yuk, H., Zhang, T., Lin, S., Parada, G. A. \& Zhao, X. Tough bonding of hydrogels to diverse non-porous surfaces. Nat. Mater. 15, 190-196 (2016).

15. Aifantis, E. C. The physics of plastic deformation. Int. J. Plasticity 3, 211-247 (1987).

16. An, S. Y., Schon, T. B., McAllister, B. T. \& Seferos, D. S. Design strategies for organic carbonyl materials for energy storage: small molecules, oligomers, polymers, and supramolecular structures. EcoMat 2, e12055 (2020).

17. Mo, F. et al. Biomimetic organohydrogel electrolytes for high-environmental adaptive energy storage devices. EcoMat 1, e12008 (2019).

\section{ACKNOWLEDGEMENTS}

This research was supported by the National Key R\&D Program of China under Project 2019YFA0705104.

\section{AUTHOR CONTRIBUTIONS}

G.L. conducted the research as a lead author and composed the paper under the supervision of Prof. Chunyi Zhi. All authors reviewed the paper and provided comments and corrections.

\section{COMPETING INTERESTS}

The authors declare no competing interests.

\section{ADDITIONAL INFORMATION}

Correspondence and requests for materials should be addressed to Chunyi Zhi.

Reprints and permission information is available at http://www.nature.com/reprints

Publisher's note Springer Nature remains neutral with regard to jurisdictional claims in published maps and institutional affiliations.

(c) Open Access This article is licensed under a Creative Commons adaptation, distribution and reproduction in any medium or format, as long as you give appropriate credit to the original author(s) and the source, provide a link to the Creative Commons license, and indicate if changes were made. The images or other third party material in this article are included in the article's Creative Commons license, unless indicated otherwise in a credit line to the material. If material is not included in the article's Creative Commons license and your intended use is not permitted by statutory regulation or exceeds the permitted use, you will need to obtain permission directly from the copyright holder. To view a copy of this license, visit http://creativecommons. org/licenses/by/4.0/.

(c) The Author(s) 2021 